\title{
O conhecimento produzido no Programa de Pós-Graduação em Enfermagem: a Enfermagem Psiquiátrica
}

\author{
THE KNOWLEDGE PRODUCED IN THE GRADUATE PROGRAM IN NURSING: \\ PSYCHIATRIC NURSING \\ EL CONOCIMIENTO PRODUCIDO EN EL PROGRAMA DE POST-GRADUACIÓN EN ENFERMERÍA: \\ LA ENFERMERÍA PSIQUIÁTRICA
}

\section{Sônia Barros ${ }^{1}$, Márcia Aparecida Ferreira de Oliveira², Ana Luisa Aranha e Silva ${ }^{3}$, Rosangela Katia de Carvalho $^{4}$, Ana Lúcia Machado ${ }^{5}$, Luciana de Almeida Colvero ${ }^{6}$, Evalda Cançado Arantes ${ }^{7}$}

\begin{abstract}
RESUMO
Após quase 30 anos de criação da Área de Concentração Enfermagem Psiquiátrica, no Programa de Pós-Graduação da Escola de Enfermagem da USP, é importante olhar a produção científica gerada para que fundamentem reflexões sobre este ensino. Este estudo é descritivo-exploratório, usou como fonte os registros do Serviço de Pós-Graduação, resumos de dissertações e teses; memorandos e ofícios; os dados coletados foram analisados à luz das transformações ocorridas na estrutura que titulou 60 alunos, dos quais 50 cursaram mestrado e 10 doutorado e que vem reestruturando suas disciplinas, linhas de pesquisa e projetos com o objetivo de atender as avaliações internas e externas e às diretrizes das agências de fomento do País. A produção científica, atualmente, evidência alinhamento com as diretrizes da Reforma Psiquiátrica e tendência de produzir conhecimentos a partir das práticas concretas dos trabalhadores nos campos da assistência, a gestão e ensino de Saúde Mental.
\end{abstract}

\section{DESCRITORES}

Enfermagem psiquiátrica.

Educação de pós-graduação em enfermagem.

Pesquisa em enfermagem.

\section{ABSTRACT}

After almost 30 years since the creation of the Psychiatric Nursing Concentration Area in the Graduate Program of the University of São Paulo Nursing School, it is important to take a look at its scientific production in order to substantiate reflection about teaching. This is a descriptive-exploratory study. It used as sources the registers of the Graduate Service, abstracts from theses and dissertations, and memoranda and official correspondence. The data collected were analyzed in the light of the transformations occurred in this structure that has graduated 60 students, of which 50 obtained a Master's Degree and $10 \mathrm{ahD}$, and that has been restructuring its disciplines, research lines and projects with the objective of responding to internal and external evaluations and adjusting to the guidelines of the country's fostering agencies. Current scientific production points out to an alignment with the guidelines of the Psychiatric Reform, and a tendency to produce knowledge through the concrete practice of the workers in the assistance field, the mana-gement and the teaching of Mental Health.

\section{KEY WORDS}

Psychiatric Nursing.

Education, Nursing, Graduate. Nursing research.

\section{RESUMEN}

Después de casi 30 años de creación del Área de Concentración de Enfermería Psiquiátrica, en el Programa de Postgrado de la Escuela de Enfermería de la USP, es importante mirar la producción científica generada que fundamenten las reflexiones sobre esta enseñanza. En este estudio descriptivo exploratorio, se usó como fuente los registros del Servicio de Postgrado, resúmenes de tesis de maestría y doctorado; memorandums y oficios; los datos recolectados fueron analizados a la luz de las transformaciones ocurridas en la estructura que graduó a 60 alumnos, de los cuales 50 cursaron maestría y 10 doctorado y que vienen reestructurando sus disciplinas, líneas de investigación y proyectos con el objetivo de atender a las evaluaciones internas y externas y a las directivas de las agencias de fomento del País. La producción científica, actualmente, evidencia alineamiento con las directrices de la Reforma Psiquiátrica y tendencia a producir conocimientos a partir de las prácticas concretas de los trabajadores en los campos de la asistencia, la gestión y la enseñanza de Salud Mental.

\section{DESCRIPTORES}

Enfermería psiquiátrica.

Educación de postgrado en enfermería.

Investigación en enfermería.
1 Professor Doutor do Departamento de Enfermagem MaternoInfantil e Psiquiátrica da Escola de Enfermagem da Universidade de São Paulo (EEUSP). Coordenadora da Área de Concentração de Enfermagem Psiquiátrica do Programa de PósGraduação da EEUSP. sobarros@usp.br

2 Professor Doutor do Departamento de Enfermagem MaternoInfantil e Psiquiátrica da EEUSP. marciaap@usp.br

3 Professor Doutor do Departamento de Enfermagem MaternoInfantil e Psiquiátrica da EEUSP. anaranha@usp.br

4 Técnico Especializado do Departamento de Enfermagem MaternoInfantil e Psiquiátrica da EEUSP. rosaenp@usp.br

5 Professor Doutor do Departamento de Enfermagem MaternoInfantil e Psiquiátrica da EEUSP. almachad@usp.br

6 Professor Doutor do Departamento de Enfermagem MaternoInfantil e Psiquiátrica da EEUSP. lucix@usp.br

7 Professor Doutor do Departamento de Enfermagem MaternoInfantil e Psiquiátrica da EEUSP 
Sônia Barros

Márcia Ap. F. de Oliveira

Ana Luisa Aranha e Silva

Rosangela K. Carvalho

Ana Lúcia Machado

Luciana A. Colvero

Evalda Cançado Arantes

\section{INTRODUÇÃO}

Decorrente da criação do Programa de Pós-Graduação, nível mestrado, na Escola de Enfermagem da Universidade de São Paulo em 1973, a Área de Concentração Enfermagem Psiquiátrica foi aprovada pela Câmara de Pós-Graduação da Universidade de São Paulo em 1975, juntamente com outras três ${ }^{(1)}$.

Desempenhou um papel decisivo na implantação do referido Programa e da área específica, a Professora Evalda Cançado Arantes que obteve seu grau de doutor em 1972 e, desde então, formou mestres e doutores, que continuam formando novos mestres e doutores, contribuindo decisivamente para a qualificação de docentes e enfermeiros e para a formação de pesquisadores.

Nesses anos de implementação, a Área de Concentração albergou docentes da casa, da especialidade, de outras especialidades, docentes de outras escolas de Enfermagem, enfermeiros de serviços e, mais recentemente, outros profissionais, para formar docentes para ensino superior, profissionais especializados e pesquisadores para a área. Desse processo resultaram estudos, traduzidos nas formas de dissertações e teses, que contribuem para a consolidação do saber em enfermagem e em saúde mental.

Após quase 30 anos de existência é importante recuperar esse percurso, para que se tenha subsídios que fundamentem as reflexões sobre o ensino da pós-graduação em enfermagem e saúde mental e seu compromisso com a produção de conhecimentos.

Este estudo foi realizado na perspectiva de reconhecer a produção científica da área e também os alunos que desenvolveram esses estudos.

\section{OBJETIVOS}

- Caracterizar o perfil dos alunos da Pós-Graduação em Enfermagem na Área de Concentração Enfermagem Psiquiátrica;

Tabela 1 - Caracterização discente da Área de Concentração Enfermagem Psiquiátrica do Programa de Pós-Graduação da Escola de Enfermagem da Universidade de São Paulo segundo década, sexo e nível: 1977-2003 - São Paulo, 2004.

\begin{tabular}{|c|c|c|c|c|c|c|}
\hline \multirow[t]{3}{*}{ Década } & \multicolumn{4}{|c|}{ Alunos } & \multicolumn{2}{|c|}{ Total } \\
\hline & \multicolumn{2}{|c|}{ Mestrado } & \multicolumn{2}{|c|}{ Doutorado } & \multirow[b]{2}{*}{$\mathbf{N}$} & \multirow[b]{2}{*}{$\%$} \\
\hline & Feminino & Masculino & Feminino & Masculino & & \\
\hline $70-79$ & 4 & - & - & - & 4 & 6,67 \\
\hline 90-99 & 17 & 2 & 6 & - & 25 & 41,67 \\
\hline 00-03 & 14 & 1 & 3 & 1 & 19 & 31,67 \\
\hline Total & 46 & 4 & 9 & 1 & 60 & 100,00 \\
\hline
\end{tabular}

Fonte: Serviço de Pós-Graduação da Escola de Enfermagem da Universidade de São Paulo/2004
- Identificar as transformações ocorridas na Área;

- Analisar os elementos da produção científica da Área, à luz destas transformações.

\section{METODOLOGIA}

Trata-se de um estudo descritivo-exploratório que se preocupa em descrever as características dos alunos, sua produção científica e explicitar aspectos que possibilitem a sua compreensão(2). Foram usadas Fontes Documentais que incluíram registros do Serviço de Pós-Graduação da Escola de Enfermagem da Universidade de São Paulo (EEUSP), resumos das Dissertações e Teses produzidos na Área, memorandos e ofícios. Os dados coletados foram tabulados e analisados à luz das transformações ocorridas na estrutura da Área de Concentração.

\section{OS ALUNOS}

Ao longo desses anos a Área de Concentração Enfermagem Psiquiátrica, nível mestrado, abriu inscrições 16 vezes. Além disso, a criação do doutorado em Enfermagem, extensão do mestrado do Programa de Pós Graduação em Enfermagem, tem titulado doutores cujos objetos de pesquisa e orientadores são da área temática da Enfermagem Psiquiátrica.

Dos 60 alunos titulados pelo Programa nesta Área de Concentração, 50 (83,3\%) deles cursou o nível mestrado e 10 (16,7\%) o nível doutorado. Destes 60 alunos, 55 (91,7\%) eram do sexo feminino e 05 $(8,3 \%)$ do sexo masculino.

Na década de 1970 a 1979 apenas 04 alunos, todos do sexo feminino, concluíram o nível mestrado. Nas décadas que se seguem há expressivo aumento no número de alunos, conforme evidencia a Tabela 1. 
Os quatro alunos do primeiro grupo de ingressantes, em 1975, defenderam suas dissertações a partir de 1977, daí o número reduzido na década de 1970.

Com relação à faixa etária, importa destacar que a grande maioria dos alunos ao defender suas dissertações e teses estava na faixa de 30 a 49 anos; foram 51 alunos, o que corresponde a $85 \%$ do total de alunos nesta Área de Concentração. Esta prevalência pode ser explicada por um dos critérios de seleção para a inscrição na Área, que era a comprovação de dois anos de experiência profissional, o que pode ser um fator de limitação para os profissionais mais jovens.

Tabela 2 - Caracterização discente da Área de Concentração Enfermagem Psiquiátrica do Programa de Pós-Graduação da Escola de Enfermagem da Universidade de São Paulo segundo faixa etária, nível e sexo: 1977-2003 - São Paulo, 2004.

\begin{tabular}{|c|c|c|c|c|c|c|}
\hline \multirow{3}{*}{$\begin{array}{c}\text { Faixa } \\
\text { etãria* }\end{array}$} & \multicolumn{4}{|c|}{ Alunos } & \multicolumn{2}{|c|}{ Total } \\
\hline & \multicolumn{2}{|c|}{ Mestrado } & \multicolumn{2}{|c|}{ Doutorado } & \multirow[b]{2}{*}{$\mathbf{N}$} & \multirow[b]{2}{*}{$\%$} \\
\hline & Feminino & Masculino & Feminino & Masculino & & \\
\hline $20-29$ & 2 & - & - & - & 2 & 3,33 \\
\hline $30-39$ & 31 & 4 & 1 & - & 36 & 60,00 \\
\hline $40-49$ & 8 & - & 6 & 1 & 15 & 25,00 \\
\hline $50-59$ & 1 & - & 2 & - & 3 & 5,00 \\
\hline Não constava & 4 & - & - & - & 4 & 6,67 \\
\hline Total & 46 & 4 & 9 & 1 & 60 & 100,00 \\
\hline
\end{tabular}

*Faixa etária correspondente ao período em que defendeu a dissertação ou a tese

Fonte: Serviço de Pós-Graduação da Escola de Enfermagem da Universidade de São Paulo/2004

Buscou-se também conhecer a ocupação dos alunos que finalizaram o Programa, relacionando este dado com as informações sobre sexo e titulação. Identificou-se que dos 60 alunos deste período, 49 $(81,67 \%)$ trabalhavam no ensino e na assistência de enfermagem, o que corresponde em números absolutos a 29 e 20 alunos, respectivamente. Dos demais alunos, 03 trabalhavam na direção de serviços, 01 não especificado e de 07 alunos não constava esta informação nos documentos consultados.

Tabela 3 - Caracterização discente da Área de Concentração Enfermagem Psiquiátrica do Programa de Pós-Graduação da Escola de Enfermagem da Universidade de São Paulo segundo ocupação e nível: 1977-2003 - São Paulo, 2004.

\begin{tabular}{lccc}
\hline \multicolumn{1}{c}{ Ocupação } & Mestrado & Doutorado & Total \\
\hline Ensino & 23 & 6 & 29 \\
Assistência & 20 & - & 20 \\
Direção de serviço & 3 & - & 3 \\
Outros & 1 & - & 1 \\
Não constava & 3 & 4 & 7 \\
\hline Total & 50 & 10 & 60 \\
\hline
\end{tabular}

Fonte: Serviço de Pós-Graduação da Escola de Enfermagem da Universidade de São Paulo/2004

Cabe destacar que daqueles 29 alunos, cuja ocupação era o ensino, 23 cursaram o mestrado e 06 o doutorado. Com relação àqueles que atuavam na assistência de enfermagem naquele momento, a totalidade era de alunos do mestrado.
O conhecimento produzido no Programa de Pós-Graduação em Enfermagem: a Enfermagem Psiquiátrica 
Sônia Barros

Márcia Ap. F. de Oliveira Ana Luisa Aranha e Silva Rosangela K. Carvalho

Ana Lúcia Machado

Luciana A. Colvero

Evalda Cançado Arantes

Tabela 4 - Caracterização discente da Área de Concentração Enfermagem Psiquiátrica do Programa de Pós-Graduação da Escola de Enfermagem da Universidade de São Paulo, segundo ocupação e período de titulação: 1977-2003 - São Paulo, 2004.

\begin{tabular}{|c|c|c|c|c|c|c|c|c|c|c|}
\hline \multirow[t]{4}{*}{ Ocupação } & \multicolumn{8}{|c|}{ Alunos } & \multicolumn{2}{|c|}{ Total } \\
\hline & \multicolumn{4}{|c|}{ Mestrado } & \multicolumn{4}{|c|}{ Doutorado } & & \\
\hline & \multicolumn{4}{|c|}{ Década } & \multicolumn{4}{|c|}{ Década } & & \\
\hline & $70-79$ & $80-89$ & $90-99$ & $00-03$ & $70-79$ & $80-89$ & $90-99$ & $00-03$ & $\mathbf{N}$ & $\%$ \\
\hline Ensino & 3 & 7 & 9 & 4 & - & - & 6 & - & 29 & 48,33 \\
\hline Assistência & - & 4 & 6 & 10 & - & - & - & - & 20 & 33,33 \\
\hline Direção de Serviço & 1 & 1 & 1 & - & - & - & - & - & 3 & 5,00 \\
\hline Outros & - & - & - & 1 & - & - & - & - & 1 & 1,67 \\
\hline Não Especificado & - & - & 2 & 1 & - & - & - & 4 & 7 & 11,67 \\
\hline Total & 4 & 12 & 18 & 16 & 0 & 0 & 6 & 4 & 60 & 100,00 \\
\hline
\end{tabular}

Fonte: Serviço de Pós-Graduação da Escola de Enfermagem da Universidade de São Paulo/2004

Nas primeiras décadas de implementação da Área de Concentração houve um predomínio de docentes entre os que se titularam, o que se justifica pela necessidade de formar quadros para os programas de Pós-Graduação das Escolas de Enfermagem. A partir da década de 1990 a proporção de enfermeiros assistenciais aumentou, o que criou uma expectativa importante, ao se considerar que esses enfermeiros estariam influenciando o campo das práticas, como multiplicadores do ensino e da pesquisa em saúde mental.

Com relação à procedência dos alunos do Programa, identificou-se que o maior contingente é da própria capital de São Paulo, num total de 35 alunos.
Destes, 31 alunos (88,57\%) defenderam o mestrado e 04 alunos (11,43\%) o doutorado. Também tivemos 01 aluno de mestrado, que depois fez o doutorado, proveniente de outro país da América Latina.

Contabilizou-se também 13 (21,66\%) alunos do interior do Estado de São Paulo. Com relação aos alunos provenientes de outros Estados do Brasil, identificou-se um total de 10 alunos (16,66\%). Chama atenção que do total de doutorandos, 04 (40\%) eram de fora do Estado de São Paulo, que se explica pelo número reduzido de Programas de Doutorado em Enfermagem no País e pela concentração destes na região sudeste.

Tabela 5 - Caracterização discente da Área de Concentração Enfermagem Psiquiátrica do Programa de Pós-Graduação da Escola de Enfermagem da Universidade de São Paulo segundo procedência e nível: 1977-2003 - São Paulo, 2004.

\begin{tabular}{lccrrr}
\hline \multicolumn{1}{c}{ Procedência } & Mestrado & Doutorado & N & Total & \% \\
\hline São Paulo - Capital & 31 & 4 & 35 & 58,33 \\
Interior de São Paulo & 12 & 1 & 13 & 21,67 \\
Outro Estado & 6 & 4 & 10 & 16,67 \\
Outro País & 1 & 1 & 2 & 3,33 \\
\hline Total & $\mathbf{5 0}$ & $\mathbf{1 0}$ & $\mathbf{6 0}$ & $\mathbf{1 0 0 \%}$ \\
\hline
\end{tabular}

Fonte: Serviço de Pós-Graduação da Escola de Enfermagem da Universidade de São Paulo;2004

Face à aposentadoria dos professores titulados, o corpo docente da Área Temática além de reduzido, contava com a maioria de docentes ainda em formação. Assim, nos anos de 2000, 2001 e 2002 não foram oferecidas vagas para abertura do processo seletivo na área de concentração, o que voltou a ocorrer em 2003 e com caráter multiprofissional.
Atualmente estão vinculadas ao Programa de Pós-Graduação, na área de Enfermagem Psiquiátrica, 18 mestrandos e 02 doutorandos em fase de elaboração de projeto e dissertação/tese ou cursando disciplinas. 


\section{O CONHECIMENTO PRODUZIDO}

Apresenta-se aqui o perfil da produção de conhecimentos gerado pelos mestres e doutores titulados pela Área de Concentração de Enfermagem Psiquiátrica.

A produção científica é fruto de condições sociais específicas de produção e vinculada a uma determinada estrutura e funcionamento do campo científico. Portanto, o universo da produção de conhecimento científico é um campo social como outro qualquer que está aqui expresso em temáticas relevantes para o cuidado e que acompanham os diversos momentos e transformações na assistência e ensino de enfermagem, nas políticas públicas da saúde mental e, também, nos paradigmas que sustentam a compreensão dos fenômenos investigados.

Para realizar a descrição, mesmo que panorâmica, da produção científica da Área de Concentração é fundamental observar as bases onde o ensino está estruturado. Até 1987 o curso estava estruturado com 03 disciplinas.

A disciplina Enfermagem Psiquiátrica (ENP 750), que tinha como objetivos que ao seu término o aluno fosse capaz de planejar e executar levantamento das condições de assistência psiquiátrica na comu- nidade e determinar os padrões de assistência de enfermagem no campo de saúde mental.

A disciplina Enfermagem Psiquiátrica III (ENP 5751), que oferecia as bases teóricas que fundamentam o relacionamento terapêutico enfermeira-paciente e o ensino do processo de relacionamento terapêutico.

A disciplina Enfermagem Psiquiátrica IV (ENP 5753), que tinha como objetivos, entre outros, que o aluno fosse capaz de demonstrar habilidade em analisar criticamente programas do curso de graduação em enfermagem.

Em 1994, o objetivo da Área de Concentração do Programa de Pós-Graduação em Enfermagem da Escola de Enfermagem da Universidade de São Paulo era “desenvolver conhecimentos específicos de enfermagem de modo a direcionar a prática científica da profissão”(3). Portanto, parece haver coerência entre o objetivo e as disciplinas ofertadas, que eram fundamentalmente instrumentais.

No período compreendido entre 1977, ano das primeiras defesas, e 2003 foram produzidas 60 pesquisas, sendo 50 (83\%) de mestrado e 10 (17\%) de doutorado de 1992 a 2003, conforme ilustra o Gráfico 1.
O conhecimento produzido no Programa de Pós-Graduação em Enfermagem: a Enfermagem Psiquiátrica

Gráfico 1 - Produção da Área de Concentração Enfermagem Psiquiátrica do Programa de Pós Graduação da Saúde Mental da Escola de Enfermagem da Universidade de

São Paulo: 1977-2003 - São Paulo, 2004.

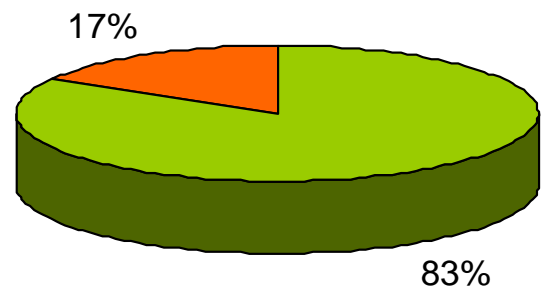

Mestrado

Doutorado

Fonte: Serviço de Pós-Graduação da Escola de Enfermagem da Universidade de São Paulo/2004

Buscou-se, por meio da leitura dos resumos, identificar os temas estudados nas dissertações e teses. Os temas foram agregados por semelhanças em categorias, e nas duas primeiras décadas podemos observar o que se segue. 
Sônia Barros

Márcia Ap. F. de Oliveira Ana Luisa Aranha e Silva Rosangela K. Carvalho

Ana Lúcia Machado

Luciana A. Colvero

Evalda Cançado Arantes

Tabela 6 - Caracterização da produção científica da Área de Conhecimento Enfermagem Psiquiátrica do Programa de Pós-Graduação da Escola de Enfermagem da Universidade de São Paulo, no mestrado, segundo década e tema 1973-1989 - São Paulo, 2004.

\begin{tabular}{lcccc}
\hline & \multicolumn{2}{c}{ Décadas } & \multicolumn{2}{c}{ Total } \\
\cline { 2 - 4 } \multicolumn{1}{c}{ TEMAS } & $70-79$ & $80-89$ & $\mathrm{~N}$ & $\%$ \\
\hline Assistência & - & 2 & 2 & 12,50 \\
Ensino de enfermagem psiquiátrica & - & 2 & 2 & 12,50 \\
Caracterização da população atendida & 4 & - & 4 & 25,00 \\
$\begin{array}{l}\text { Percepção dos atores envolvidos na assistência } \\
\text { Representação do pessoal de enfermagem e das }\end{array}$ & - & 6 & 6 & 37,50 \\
pessoas com Transtorno Mental sobre a loucura & - & 1 & 1 & 6,25 \\
Temas fora da área & - & 1 & 1 & 6,25 \\
\hline Total & 4 & 12 & 16 & 100,00 \\
\hline
\end{tabular}

Fonte: Dissertações defendidas no período, disponíveis na Biblioteca da Escola de Enfermagem da Universidade de São Paulo.

Dentre os 16 estudos realizados, entre 1975 e 1989, 06 (37,50\%) relacionam-se às percepções, sentimentos e opiniões da equipe de enfermagem e dos familiares sobre as manifestações de comportamento das pessoas com transtorno mental e definem como objetivo planejar a assistência de enfermagem. Outros 02 estudos são explicitamente sobre assistência de enfermagem, totalizando assim 08 estudos $(50,00 \%)$ sobre tema nessa categoria. $\mathrm{Na}$ década de 1980 um único estudo que trabalha com representações sociais foi orientado por professor de outra Área de Concentração.

As linhas de pesquisa registradas nos relatórios da Coordenação de Aperfeiçoamento de Pessoal de Nível Superior (CAPES) pela Área de Concentração em 1988, segundo Paula, Koizumi i( ${ }^{(4)}$, eram as seguintes: Assistência de Enfermagem; Comunicação em Enfermagem; Ensino e Aprendizagem em Enfermagem; Metodologia de Pesquisa em Enfermagem. Portanto, as pesquisas realizadas no período em análise concentravam-se nas linhas da Assistência e do Ensino e os demais estudos eram independentes.

Em estudo sobre a pesquisa de enfermagem no Brasil, verificou-se que nas décadas de 1970 a 79, os estudos referentes aos processos assistenciais e necessidades do paciente representam as preocupações dos enfermeiros e que o mesmo aconteceu na década seguinte ${ }^{(5)}$.

A produção na Área de Enfermagem Psiquiátrica, no mesmo período, foi estudada por outra auto$\mathrm{ra}^{(6)}$, que a caracterizou como restrita e, em sua maioria, direcionada ao levantamento de problemas.

Ressalte-se que na década de 1980 importantes transformações ocorriam nas diretrizes políticas na área de saúde mental. No Estado de São Paulo propunha-se a mudança do modelo assistencial, hegemonicamente hospitalar, para a ampliação do número de serviços ambulatoriais. Tais serviços tornaram-se um campo novo de prática para os enfermeiros. A organização dos trabalhadores de saúde mental e a realização de vários eventos onde se discutia tais transformações deu origem ao movimento denominado de Reforma Psiquiátrica. As dissertações ora analisadas evidenciam que os pesquisadores ainda não indicavam os problemas relacionados à reorientação do modelo e das práticas assistenciais como objeto de estudo e os cenários das pesquisas eram, predominantemente o hospital, em especial o hospital psiquiátrico. 
Tabela 7- Caracterização da Produção Científica da Área de Concentração Enfermagem Psiquiátrica do Programa de Pós-Graduação em Enfermagem da Escola de Enfermagem da Universidade de São Paulo, segundo campo de pesquisa e sujeito informante, no período de 1973-1989 - São Paulo, 2004.

\begin{tabular}{|c|c|c|c|c|c|c|c|c|c|}
\hline \multirow[t]{2}{*}{ Local } & \multicolumn{7}{|c|}{ Sujeitos } & \multicolumn{2}{|c|}{ Total } \\
\hline & $\begin{array}{l}\text { Aluno } \\
\text { Enferm. }\end{array}$ & Enfermeiro & $\begin{array}{l}\text { Equipe de } \\
\text { Enfermagem }\end{array}$ & $\begin{array}{l}\text { Equipe de } \\
\text { Enfermagem } \\
\text { e Paciente }\end{array}$ & $\begin{array}{l}\text { Pacientes } \\
\text { Familiares }\end{array}$ & Família & Paciente & $\mathrm{N}$ & $\%$ \\
\hline Ambulatório & & & & & & & & & \\
\hline $\begin{array}{l}\text { Saúde Mental } \\
\text { Escola de }\end{array}$ & & & & & & 1 & & 1 & 6,25 \\
\hline Enfermagem & 1 & & & & & & & 1 & 6,25 \\
\hline $\begin{array}{l}\text { Hospital Geral } \\
\text { Hospital }\end{array}$ & & 4 & 1 & & & & & 5 & 31,25 \\
\hline Psiquiátrico & & & 2 & 1 & 1 & 3 & 2 & 9 & 56,25 \\
\hline Total & 1 & 4 & 3 & 1 & 1 & 4 & 2 & 16 & 100,00 \\
\hline
\end{tabular}

Fonte: Dissertações defendidas no período, disponíveis na Biblioteca da Escola de Enfermagem da Universidade de São Paulo.

Com relação à metodologia, trata-se de estudos descritivos e um único estudo explicitou o referencial teórico-metodológico que foi a fenomenologia. A análise foi predominantemente quantitativa correspondendo a $75 \%$ dos trabalhos.

\section{O PROCESSO DE REESTRUTURAÇÃO DA ÁREA DE CONCENTRAÇÃO A PARTIR DA DÉCADA DE 90}

A partir da década de 1990 agregam-se à produção da Área, as teses defendidas no Doutorado em Enfermagem orientadas por docentes da Área Temática Enfermagem Psiquiátrica. Ampliam-se os temas, mas ainda prevalecem aqueles relacionados à categoria de Assistência.

Tabela 8 - Caracterização da produção das teses e dissertações da Área de Concentração Enfermagem Psiquiátrica do Programa de Pós-Graduação da Escola de Enfermagem da Universidade de São Paulo, no mestrado, segundo década e tema 1990-2003 - São Paulo, 2004.

\begin{tabular}{|c|c|c|c|c|c|c|}
\hline \multirow{3}{*}{ Temas } & \multicolumn{4}{|c|}{ Décadas } & \multicolumn{2}{|c|}{ Total } \\
\hline & \multicolumn{2}{|c|}{$90-99$} & \multicolumn{2}{|c|}{$00-03$} & \multirow{2}{*}{$\mathbf{N}$} & \multirow{2}{*}{$\%$} \\
\hline & $\mathbf{M}$ & D & $\mathbf{M}$ & $\mathbf{D}$ & & \\
\hline Assistência & 6 & 2 & 7 & 1 & 16 & 36,36 \\
\hline Ensino de Enfermagem & 2 & - & 4 & 2 & 8 & 18,18 \\
\hline Funções do Enfermeiro & 5 & - & - & - & 5 & 11,36 \\
\hline Percepção dos Atores Envolvidos & 2 & - & 2 & 1 & 5 & 11,36 \\
\hline Política de Saúde Mental & 1 & - & - & - & 1 & 2,28 \\
\hline Organização de Serviço & - & - & 1 & - & 1 & 2,28 \\
\hline Temas fora da Área & 3 & 4 & 1 & - & 8 & 18,18 \\
\hline Total & 19 & 6 & 15 & 4 & 44 & 100,00 \\
\hline
\end{tabular}

Fonte: Dissertações defendidas no período, disponíveis na Biblioteca da Escola de Enfermagem da Universidade de São Paulo.

É possível observar novas temáticas, como os estudos sobre as funções do enfermeiro psiquiátrico, as políticas de saúde mental e organização de serviço. A leitura dos resumos sugere que essas temáticas estão relacionadas às questões e transformações que a Reforma Psiquiátrica trouxe para o campo de trabalho do enfermeiro. Problematizam-se também as transformações necessárias no ensino, estratégias para reduzir o "medo" dos alunos que cursam enfermagem psiquiátrica na graduação e para contemplar o fazer no novo paradigma da saúde mental.

Também é possível observar que temas relacionados à outras áreas do conhecimento têm um alto percentual, o que se explica pelo acolhida aos docentes, professores da EE de outras especialidades, que necessitavam se titular.
O conhecimento produzido no Programa de Pós-Graduação em Enfermagem: a Enfermagem Psiquiátrica 
Sônia Barros

Márcia Ap. F. de Oliveira Ana Luisa Aranha e Silva Rosangela K. Carvalho Ana Lúcia Machado Luciana A. Colvero Evalda Cançado Arantes
Em 1998 iniciou-se um processo de alinhamento das Linhas de Pesquisa, disciplinas oferecidas e projetos desenvolvidos pelos docentes com o objetivo de atender às diretrizes das agências de fomento do País. De acordo com o Relatório de Linhas e Projetos de Pesquisa da Comissão de Pesquisa EEUSP, em 1999 a Área de Concentração de Enfermagem Psiquiátrica tinha sete linhas definidas:

1. Aspectos psicossociais do processo saúde/ doença;

2. Gestão da qualidade em saúde;

3. Ensino e Aprendizagem do aluno;

4. Assistir/cuidar em enfermagem;

5. Ensino - Aprendizagem do profissional;

6. Desenvolvimento Tecnológico na Saúde;

7. Comunicação em enfermagem.

Pode-se entender que a maioria dessas linhas era emergente porque os estudos continuavam concentrados em linhas consolidadas: Assistir/cuidar em enfermagem e Ensino e Aprendizagem do aluno.

Nesse período estava em curso um processo de reestruturação da Área de Concentração de Enfer-magem Psiquiátrica. Devido à aposentadoria de docentes e para atender as reformulações necessárias no Programa de Pós-Graduação da Escola de Enfermagem da Universidade de São Paulo, face às avaliações internas e externas, em 1998, foram desativadas disciplinas e oferecidas outras, configurando um instrumental de base conceitual e menos tecnológico:

Tabela 9 -Caracterização da Produção Científica da Área de Concentração Enfermagem Psiquiátrica do Programa de Pós-Graduação da Escola de Enfermagem da Universidade de São Paulo, segundo campo da pesquisa e sujeitos informantes, no período de 1990-2003 - São Paulo, 2004.

\begin{tabular}{|c|c|c|c|c|c|c|c|c|}
\hline \multirow[t]{2}{*}{ Local } & \multirow[b]{2}{*}{$\begin{array}{l}\text { Alunos } \\
\text { Enfermagem }\end{array}$} & \multirow[b]{2}{*}{ Docente } & \multirow[b]{2}{*}{ Enfermeiro } & \multirow[b]{2}{*}{$\begin{array}{l}\text { Equipe de } \\
\text { Enfermagem }\end{array}$} & \multirow[b]{2}{*}{ Família } & \multirow[b]{2}{*}{ Paciente } & \multicolumn{2}{|c|}{ Total } \\
\hline & & & & & & & $\mathrm{N}$ & $\%$ \\
\hline Ambulatório Geral & & & & 1 & & 2 & 3 & 6,82 \\
\hline Ambulatório Hospital & & & & & & & & \\
\hline Psiquiátrico & & & & & 1 & 1 & 2 & 4,55 \\
\hline Serviço extra-hospitalar & & & 5 & & 1 & 2 & 8 & 18,18 \\
\hline Associação de & & & & & & & & \\
\hline Familiares & & & & & 1 & & 1 & 2,27 \\
\hline Clínica Geriátrica & & & & & & 1 & 1 & 2,27 \\
\hline Enfermaria Psiquiátrica & & & & & & & & \\
\hline em Hospital Geral & & & 1 & & & 1 & 2 & 4,55 \\
\hline Escola Ensino & & & & & & & & \\
\hline Fundamental, Médio e & & & & & & & & \\
\hline Universitário & 6 & 4 & & & & & 10 & 22,73 \\
\hline Hospital Geral & & & 5 & 1 & & 1 & 7 & 15,91 \\
\hline Hospital Psiquiátrico & & & 3 & 3 & & 3 & 9 & 20,45 \\
\hline Pronto Socorro em & & & & & & & & \\
\hline Hospital Geral & & & & 1 & & & 1 & 2,27 \\
\hline Total & 6 & 4 & 14 & 6 & 3 & 11 & 44 & $100 \%$ \\
\hline
\end{tabular}

1. ENP 5766 - Evolução histórico-social da assistência em saúde mental;

2. ENP 5767 - Tendências da assistência em saúde mental e suas influências na enfermagem;

3. ENP 5768 - Ações terapêuticas em enfermagem psiquiátrica.

Em 2000, segundo os dados do Relatório de Linhas e Projetos de Pesquisa da Comissão de Pesquisa EEUSP e na seqüência do processo de reestruturação, com as recomendações da CAPES, a área permanecia, basicamente, com as linhas de Pesquisa já citadas, acrescida de uma nova, a "Bases teóricas e conceituais em enfermagem” tendo sido retirada a linha "Desenvolvimento Tecnológico na Saúde”.

A partir de abril de 2003, os dados registrados no Relatório de Linhas e Projetos de Pesquisa da Comissão de Pesquisa EEUSP informam a alteração na designação da Área de Concentração para Saúde Mental, que está em fase de análise a aguarda aprovação da Câmara de Pós-Graduação da USP. As atuais linhas de pesquisa da Área são:

1. O processo de cuidar no campo psiquiátrico e no campo da saúde mental

2. Políticas e práticas em saúde mental e em enfermagem

E as disciplinas que sustentam o ensino da Área Temática são:

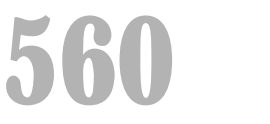

Rev Esc Enferm USP 2005; 39(Esp.):553-63.

Fonte: Dissertações defendidas no período, disponíveis na Biblioteca da Escola de Enfermagem da Universidade de São Paulo. 
1. ENP 5766 - Evolução histórico-social da assistência em saúde mental;

2. ENP 5819 - Reabilitação Psicossocial: Teorias e Práticas

3. ENP 5815 - Dimensões da Subjetividade no Cuidado em Saúde Mental

O objetivo de tais alterações é a construção de conhecimentos para o desenvolvimento de saberes e práticas que recomponham as políticas, o processo de trabalho e o ensino na área de saúde mental, considerando a interface das subjetividades nas dimensões individual e coletiva.
Com as alterações empreendidas a partir de 1998 e consolidadas em 2003, evidencia-se uma ampliação dos campos para as pesquisas assim como a diversidade dos sujeitos de estudo. Destaca-se que os serviços extra-hospitalares tornaram-se relevantes como cenários das pesquisas e as pessoas com transtornos mentais - os pacientes - passaram a ser sujeitos falantes, atores com voz.

Os níveis de pesquisa também passaram a ser mais diversificados e os estudos exploratórios tornaram-se numericamente superiores aos descritivos.

Tabela 10 - Caracterização da produção de conhecimento da Área de Concentração Enfermagem Psiquiátrica do Programa de Pós-Graduação da Escola de Enfermagem da Universidade de São Paulo, segundo nível e método, no período de 1990-2003 - São Paulo, 2004.

\begin{tabular}{lccrr}
\hline \multicolumn{1}{c}{ Método } & Dissertações & Teses & Total & \% \\
\hline Descritivo & 17 & 2 & 19 & 43,18 \\
Descritivo e exploratório & 2 & 1 & 3 & 6,82 \\
Explicativo & 1 & 0 & 1 & 2,27 \\
Exploratório & 14 & 7 & 21 & 47,73 \\
\hline Total & $\mathbf{3 4}$ & $\mathbf{1 0}$ & $\mathbf{4 4}$ & $\mathbf{1 0 0 , 0 0}$ \\
\hline
\end{tabular}

Fonte: Dissertações defendidas no período, disponíveis na Biblioteca da Escola de Enfermagem da Universidade de São Paulo.

Com relação às correntes de pensamento filosófico é possível observar na Tabela 11 uma tendência à busca de fundamentação teórica na Fenomenologia e nas Representações Sociais. No entanto, 28 (63,63\%) não explicitaram seus referenciais, o que ratifica a crítica de Silva, $\operatorname{Ramos}^{(7)}$ ao analisarem os resumos de 376 teses de doutorado defendidas no período de 1972 a 1999, onde ocorreu o mesmo fenômeno.

Tabela 11 - Caracterização da produção de conhecimento da Área de Concentração Enfermagem Psiquiátrica do Programa de Pós-Graduação da Escola de Enfermagem da Universidade de São Paulo, segundo nível e referencial teórico metodológico, no período de 1990-2003 - São Paulo, 2004.

\begin{tabular}{lcccc}
\hline \multicolumn{1}{c}{$\begin{array}{c}\text { Referencial teórico } \\
\text { metodológico }\end{array}$} & Dissertações & Teses & N & Total \\
\hline Etnografia & 1 & 4 & 5 & 11,36 \\
Fenomenologia & 2 & 3 & 5 & 11,36 \\
Materialismo histórico dialético & 1 & 0 & 1 & 2,27 \\
Teoria das representações sociais & 5 & 0 & 5 & 11,36 \\
Sem referencial teórico & 25 & 3 & 28 & 63,64 \\
\hline Total & 34 & $\mathbf{1 0}$ & $\mathbf{4 4}$ & $\mathbf{1 0 0 \%}$ \\
\hline
\end{tabular}

Fonte: Dissertações defendidas no período, disponíveis na Biblioteca da EscoladeEnfermagem da Universidade de São Paulo.

Observa-se um incremento nas pesquisas qualitativas, como demonstra a Tabela 12. 
Sônia Barros

Márcia Ap. F. de Oliveira Ana Luisa Aranha e Silva Rosangela K. Carvalho

Ana Lúcia Machado

Luciana A. Colvero

Evalda Cançado Arantes

Tabela 12 - Caracterização da produção de conhecimento da Área de Concentração Enfermagem Psiquiátrica do Programa de Pós-Graduação da Escola de Enfermagem da Universidade de São Paulo segundo década, nível e metodologia, no período de 1990-2003 - São Paulo, 2004.

\begin{tabular}{|c|c|c|c|c|c|c|}
\hline \multirow[t]{3}{*}{ Metodologia } & \multirow{2}{*}{\multicolumn{2}{|c|}{$\begin{array}{l}\text { Mestrado } \\
\text { Década }\end{array}$}} & \multirow{2}{*}{\multicolumn{2}{|c|}{$\begin{array}{l}\text { Doutorado } \\
\text { Década }\end{array}$}} & \multirow{2}{*}{\multicolumn{2}{|c|}{ Total }} \\
\hline & & & & & & \\
\hline & $90-99$ & $00-03$ & $90-99$ & 00-03 & $\mathbf{N}$ & $\%$ \\
\hline Quantitativa & 4 & 3 & 0 & 0 & 7 & 15,91 \\
\hline Qualitativa & 10 & 13 & 5 & 2 & 30 & 68,18 \\
\hline \multicolumn{7}{|l|}{ Quanti e } \\
\hline Qualitativa & 3 & 1 & 1 & 2 & 7 & 15,91 \\
\hline Total & 17 & 17 & 6 & 4 & 44 & 100,00 \\
\hline
\end{tabular}

Fonte: Dissertações defendidas no período, disponíveis na Biblioteca da Escola de Enfermagem da Universidade de São Paulo.

O Mestrado em Enfermagem Psiquiátrica do Programa de Pós-Graduação da Escola de Enfermagem da Universidade de São Paulo não tem extensão para o Doutorado, mas as docentes da Área de Concentração se responsabilizam pela orientação dos Projetos de Pesquisa do Curso de Doutorado do PPGE, cuja temática e objeto de estudo lhe concerne. O período em análise evidencia que a metodologia quantitativa pura não foi utilizada no Nível Doutorado e a maioria dos pesquisadores optou pela perspectiva qualitativa, revelando um produto coerente com o processo de alinhamento das bases teóricas que vinha ocorrendo no interior da Área de Concentração.

\section{SÍNTESE: PESQUISADORES E SEUS PRODUTOS NA ÁREA DE CONCENTRAÇÃO ENFERMAGEM PSIQUIÁTRICA}

As comemorações de 30 anos do Programa de Pós-Graduação da Escola de Enfermagem da USP mobi-lizam a necessária reflexão sobre o conhecimento produzido nessas décadas. A área de Concentração Enfermagem Psiquiátrica titulou sessenta (60) profissionais, nesse período, sendo cinqüenta (50) mestres e dez (10) doutores o que demonstra que a Área é referência para a qualificação de profissionais e formação de pesquisadores no campo de saúde mental.
A análise da produção científica do período compreendido entre 1975 e de 2003 revela que transformações têm ocorrido tanto no que diz respeito ao produto final como, também, à estrutura da área, atendendo às avaliações internas e externas e às diretrizes das agências de fomento.

Vários são os desafios que se apresentam: a autonomia deste campo de conhecimento enquanto área específica na Pós-Graduação, a extensão para o nível de doutorado, a ampliação do quadro de docentes e a sua contínua qualificação, a manutenção do compromisso com a produção de um conhecimento emancipador e a necessária redução do tempo de titulação.

Os docentes envolvidos no ensino de pós-graduação têm direcionado esforços no sentido de desenvolverem, junto aos diversos especialistas que compõem o quadro de alunos da Área, uma visão analítica do campo de Saúde Mental e uma conseqüente produção científica que possa intervir na realidade concreta dos processos de assistir, ensinar, gerenciar e pesquisar.

Compreender a ciência enquanto prática social de conhecimento é como ter uma tarefa que vai se cumprindo em diálogo com o mundo e que é afinal fundada nas vicissitudes, nas opressões e nas lutas que o compõem e a nós ${ }^{(8)}$. 
(1) Kamiyama Y. Discurso da sessão de instalação. In: Anais do Simpósio "15 anos de Pós-Graduação na Escola de Enfermagem da Universidade de São Paulo: reflexos e perspectivas”; 1988 out. 24-25; São Paulo. São Paulo: EEUSP; 1990. p. 21.

(2) Gil AC. Como elaborar projetos de pesquisa. São Paulo: Atlas; 1991.

(3) Moriya TM, Rodrigues ARF, Almeida MCP, Ruffino MC, Oliveira MHP, organizadoras. Pós-graduação "stricto sensu" em enfermagem: um estudo do seu desenvolvimento no Brasil. Ribeirão Preto: Fundação Instituto de Enfermagem de Ribeirão Preto; 1998.

(4) Paula NS, Koizumi MS. Sessão Wanda Horta: perspectiva da enfermagem e a pós-graduação. In: Anais do Simpósio “15 anos de Pós-Graduação na Escola de Enfermagem da Universidade de São Paulo: reflexos e perspectivas”; 1988 out. 24-25; São Paulo. São Paulo: EEUSP; 1990. p. 89.
(5) Cianciarullo TI, Salzano SDT. A enfermagem e a pesquisa no Brasil. Rev Esc Enferm USP 1991; 25(2):195213.

(6) Arantes EC. Pesquisa em enfermagem psiquiátrica. In: Anais do $3^{\circ}$ Seminário Nacional de Pesquisa em Enfermagem, 1984 abr. 3-6; Florianópolis. Florianópolis: ABEn; 1984. p. 4-42.

(7) Silva AL, Ramos FRS. As linhas epistemológicas do conhecimento científico. In: Anais do $10^{\circ}$ Seminário Nacional de Pesquisa em Enfermagem, 2001 maio 2730; Belém. [CD-ROM] Belém: ABEn; 2001.

(8) Santos BS. Introdução a uma ciência pós moderna. Rio de Janeiro: Graal; 1989.
O conhecimento produzido no Programa de Pós-Graduação em Enfermagem: a Enfermagem Psiquiátrica
Correspondência: Sônia Barros Rua dos Franceses, 498 Bloco Diderot - Ap. 254 Morro dos Ingleses - São Paulo 01329-001 - SP 